\title{
ATUAÇÃO DO FARMACEUTICO NA ORIENTAÇÃO AO USO DA TOXINA BOTULÍNICA
}

\author{
Isabella de Lima Oliveira Santos ${ }^{1}$ \\ Leonardo Guimarães de Andrade ${ }^{2}$ \\ Livia Cabral Lobo Andrade 3 \\ Michel Santos da Silva Andrade 4
}

RESUMO: O aumento da expectativa de vida no país tem atraído coletivamente maior atenção pela população, e seu bem-estar, incluindo autoestima e estética corporal e facial. Além disso, há também a busca pelos chamados padrões de beleza impostos pela sociedade. De acordo com os dados anuais da International Society of Aesthetic Plastic Surgery (ISAPS) em 2017, o Brasil foi o terceiro país em número de procedimentos estéticos não cirúrgicos, a aplicação de botox sendo o primeiro procedimento mais realizados. A aplicação da toxina botulínica no campo da beleza começou na década de 1990 e foi aprovada pela ANVISA para tratar rugas dinâmicas no Brasil em 2000, comercializada Marca Botox ${ }^{\circledR}$. A marca Dysport foi aprovada em 2003 e a marca foi aprovada em 2005 foi a Prosigne. Desde então, a demanda e a aplicação desse método só aumentaram. O objetivo do presente trabalho é buscar validade e segurança da aplicação da toxina butolinica para tratamento de rugas dinâmicas e também a prevenção das mesmas, para fins estéticos.

Palavras chaves: Toxina butolínica. Estética. Aplicação. Rugas.

ABSTRACT: The increase in life expectancy in the country has collectively attracted greater attention by the population, and their well-being, including self-esteem and body and facial aesthetics. In addition, there is also the search for the so-called standards of beauty imposed by society. According to annual data from the International Society of Aesthetic Plastic Surgery (ISAPS) in 2017, Brazil was the third country in the number of non-surgical cosmetic procedures, the application of botox being the first most performed procedure. The application of botulinum toxin in the field of beauty began in the 199os and was approved by ANVISA to treat dynamic wrinkles in Brazil in 200o, marketed as the Botox ${ }^{\circledR}$ brand. The Dysport ${ }^{\circledR}$ brand was approved in 2003 and the brand that was approved in 2005 was the Prosigne ${ }^{\circledR}$. Since then, the demand and application of this method has only increased. The objective of the present work is to seek validity and safety of the application of butolinic toxin for the treatment of dynamic wrinkles and also their prevention, for aesthetic purposes.

Keywords: Butolinic toxin. Aesthetics. Application. Wrinkles.

\footnotetext{
${ }^{\mathrm{I}}$ Universidade Iguaçu. Faculdade de Ciências Biológicas E Da Saúde. Curso De Graduação Em Farmácia. E-Mail: Isabella.lima98@outlook.Com.

${ }^{2}$ Orientador .Universidade Iguaçu.

${ }^{3}$ Co-Orientador. Universidade Iguaçu.

${ }^{4}$ Co-Orientador. Universidade Iguaçu.
} 


\section{INTRODUÇÃO}

A toxina botulínica, desde o século 19 vem sendo estudada e no atual século é muito reconhecida nas marcas: Botox, Dysport e Prosigne, como TXB-A aprovadas pela ANVISA no Brasil para tratamentos estéticos.

IMAGEM r: Marcas da Toxina butolínica

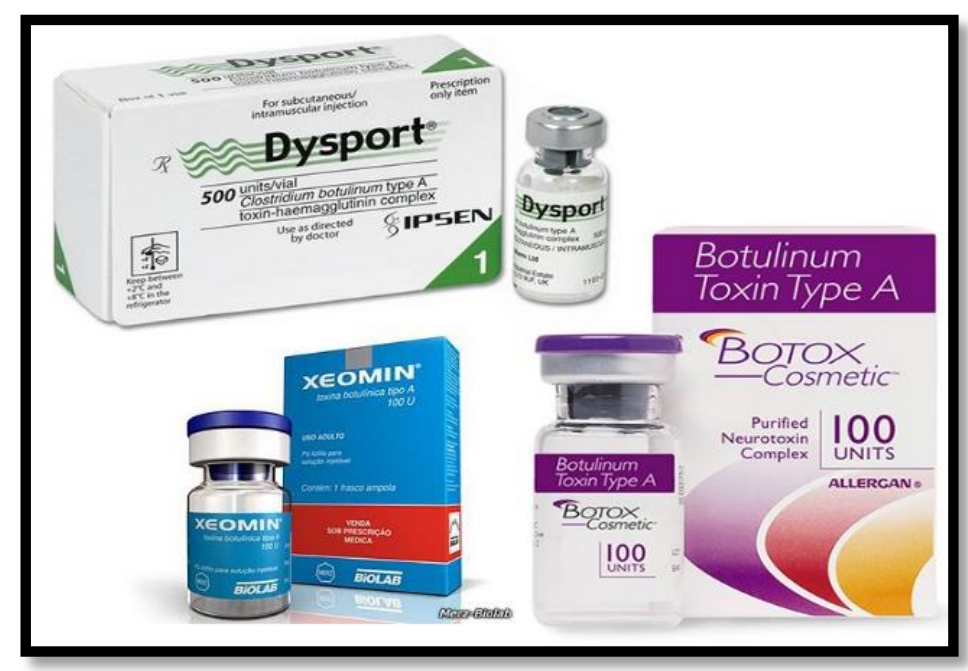

FONTE: https://camilalatorre.com.br/6-melhores-marcas-de-toxina-botulinica-no-brasil/

Toxina botulínica tipo A é amplamente estudada por Porstz, Johnson e funcionários há mais de 30 anos, inicialmente em Fort Detrick e depois na Universidade de Wisconsin, onde pela primeira vez foi produzida em laboratório (SPOSITO, MARIA).

Caracterizada como um agente biológico produzido em laboratório, a toxina botulínica do tipo A é uma substancia cristalina estável, liofilizada em albumina humana, sendo apresentada em frasco a vácuo, para ser diluída em solução salina (SPOSITO, 2004).

Naturalmente a toxina botulínica produzida pelo Clostridium botulinum, que é uma bactéria anaeróbia, e é o responsável pela produção de oito tipos sorológicos de toxina, inclusive a do tipo A, que caracteriza a toxina botulínica, e é a mais potente, sendo assim, é usada clinicamente (SPOSITO, MARIA. 2004).

“O TBA é uma terapia cosmética, eficaz para suavizar as imperfeições do rosto. $\mathrm{O}$ TBA é uma grande assistência em tratamentos de beleza porque é uma droga aplicada por profissionais, atinge níveis de segurança e resultando na satisfação do paciente" (RIBEIRO, SANTOS, GONÇALVES e CRUZ, 2014, p33). 


\section{HISTÓRICO DA TOXINA BOTULÍNICA}

O histórico da Toxina Botulínica apresenta:

- Muller e Kerner descrevem a doença botulismo na Alemanha

- Na Bélgica em I895 Ermangem consegue isolar o Clostridium botulinum

- Em igoo um surto de botulismo destruiu uma indústria de alimentos enlatados nos EUS

- Sommer isola a neurotoxina em 1920 na Universidade de Califórnia

- Em 1943 durante a II guerra Mundial ouve riscos de armas biológicas com a toxina botulínica, Profs. E. B. Fred e Ira Baldwin da Universidade de Winconsin e de Stanhope Bayne-Jones da Universidade de Yale formam um laboratório, batizado de Fort Detrick para investigar os danos e pesquisar meios de proteção contra esta bactéria

- Schantz e Dr. Erik A. Johnson em associação com o Dr. Dr. Carl Lamanna, desenvolvem e purificam a toxina na forma cristalina em 1946

- É sugerido o uso para tratamento de hiperfunção muscular por Brooks

- Dr. Alan B. Scott começa pesquisa sobre o tratamento do estrabismo no início dos anos 70

- $\quad$ Em 1977 e 1978 Scott inicia experimentos em humanos

- Schantz prepara a toxina cristalina do tipo A e submete ao Food and Drug Administration em 1979

- $\quad 1989$ a toxina botulínica do tipo A é aprovada pelo FDA como segura e eficiente para o tratamento dos distúrbios do movimento

- $\quad 1990$ o consenso do National Institutes of Health incluiu a toxina botulínica na lista de medicamentos seguros e eficientes.

\section{O QUE É A TOXINA BUTOLÍNICA?}

"As neurotoxinas do Clostridium são produzidas inicialmente como uma cadeia peptídica simples de $150 k \mathrm{Da}$ composta por 3 porções de $50 k \mathrm{Da}$ cada, a saber: L, Hc, ze Hn, 
e tem papéis no processo de intoxicação celular e bloqueio funcional" (SPOSITO, 2004, p3).

“A toxina botulínica do tipo A é uma substância neurotóxica em atual destaque, devido a sua eficácia em aplicações estéticas terapêuticas, preventivas e corretivas, não cirúrgicas, com rara resposta imunológica, com relação às rugas dinâmicas” (RIBEIRO, SANTOS, GONÇALVES e CRUZ, 2014, p33).

Essas rugas são produzidas em termos gerais por contrações repetitivas dos músculos faciais e pelo envelhecimento da pele humana, através de um mecanismo de ação eficiente e minimamente invasiva, a TBA atua transportando benefícios reais, mesmo dentro das expectativas (SPOSITO, MARIA. 2004).

Em I822, a toxina botulínica foi considerada veneno, hoje em dia é vista como terapêutica e muito usada no meio estético. Quando injetada por via muscular, essa droga tem como mecanismo de ação se ligar aos receptores terminais que ficam nos nervos motores, e isso gera um bloqueio na condução neuromuscular e consequentemente inibe a recepção da acetilcolina, assim, produzindo paralisia muscular localizada, por denervação química temporária e também inibição competitiva (UEBEL, MARJORIE. 2019).

Porém, novos receptores para a acetilcolina são repostos e assim o processo se reverte, isso traz segurança a todo o processo e ao organismo, pois a ação do TBA não atinge o Sistema Nervoso Central, não ocorro bloqueio de liberação da acetilcolina. Isso torna o processo tanto reversível como reaplicável, e sem efeitos colaterais. Sendo feito por profissionais especializados, o processo pode ser feito ao longo de anos, sem risco à saúde do paciente. $O$ protocolo para reaplicação geralmente é de seis em seis meses e de acordo com cada individualidade (SPOSITO, 2004).

"O efeito da TBA pode ser potencializado por antibióticos aminoglicosídicos ou qualquer outra droga que interfira com a transmissão neuromuscular individualidade" (SPOSITO, 2004, pri).

“Os primeiros efeitos são percebidos entre $3^{\text {a }}$ a $7^{\text {a }}$ semana na região aplicada com duração média de 3 a 6 meses, com reavaliação possível em 4 meses para reaplicação" (RIBEIRO, SANTOS, GONÇALVES e CRUZ, 20I4, p4). 


\section{MECANISMO DE AÇÃO}

A toxina botulínica basicamente inibe a liberação de exocitose da acetilcolina nas terminações nervosas motoras causando redução da contração. Esse recurso o torna muito útil clínica e terapeuticamente em uma série de casos de contração muscular excessiva.

O uso da toxina botulínica em problemas que acompanham distúrbios do movimento tem se mostrado benéfico em outros aspectos clínicos, como o alívio das situações de dor concomitantes (UEBEL, MARJORIE. 2019).

As reações adversas e aquelas distantes do ponto de injeção não induzem apenas raciocínio clínico usado em outras situações, como alterações de glândulas e músculo liso, mas forçou a pesquisa a se estender para o mecanismo de ação que pode estar envolvido.

\section{TOXINA BUTOLÍNICA APLICADA NOS MUSCÚLOS FACIAIS}

A toxina botulínica é uma proteína produzida pelo Clostridium botulinum. Quando administrado por via oral em grandes quantidades, ele bloqueia os sinais nervosos do cérebro para os músculos, resultando em uma paralisia sistêmica chamada botulismo. No entanto, ao injetar uma quantidade muito pequena do medicamento em um músculo facial específico, apenas o impulso que guia esse músculo é bloqueado, resultando em relaxamento local. Desta forma, a toxina botulínica pode bloquear o tecido muscular subjacente de linhas indesejadas.

O tratamento envolve a injeção de pequenas quantidades de medicamento nos músculos subjacentes para imobilizá-los. Os tratamentos atuais são bem tolerados, rápidos e apresentam pouquíssima recuperação. Alguns efeitos colaterais duram cerca de 3 a 7 dias após a operação. A toxina começa a fazer efeito após 7 a I4 dias, e esse efeito dura cerca de 3 a 6 meses, até que a atividade muscular seja retomada e desapareça gradualmente (UEBEL, MARJORIE. 2019).

\section{INDICAÇÃO}

A toxina botulínica é usada para suavizar linhas de expressão e rugas profundas. Por exemplo, as linhas verticais entre as sobrancelhas; os pés de galinha nos cantos dos olhos; as linhas horizontais na testa e a faixa do platisma são chamadas de pescoços de peru. Também é usado para reposicionar as sobrancelhas: os músculos ficam enfraquecidos e 
relaxados para evitar a contração. Este tratamento evita a formação de novas rugas. Alguns músculos não podem ser tratados porque desempenham um papel importante na expressão natural de uma pessoa. É o caso dos músculos que erguem as sobrancelhas e dos músculos da linha do sorriso, porque são necessários para a expressão da região e até para a alimentação.

IMAGEM 2: Tratamento para suor excessivo

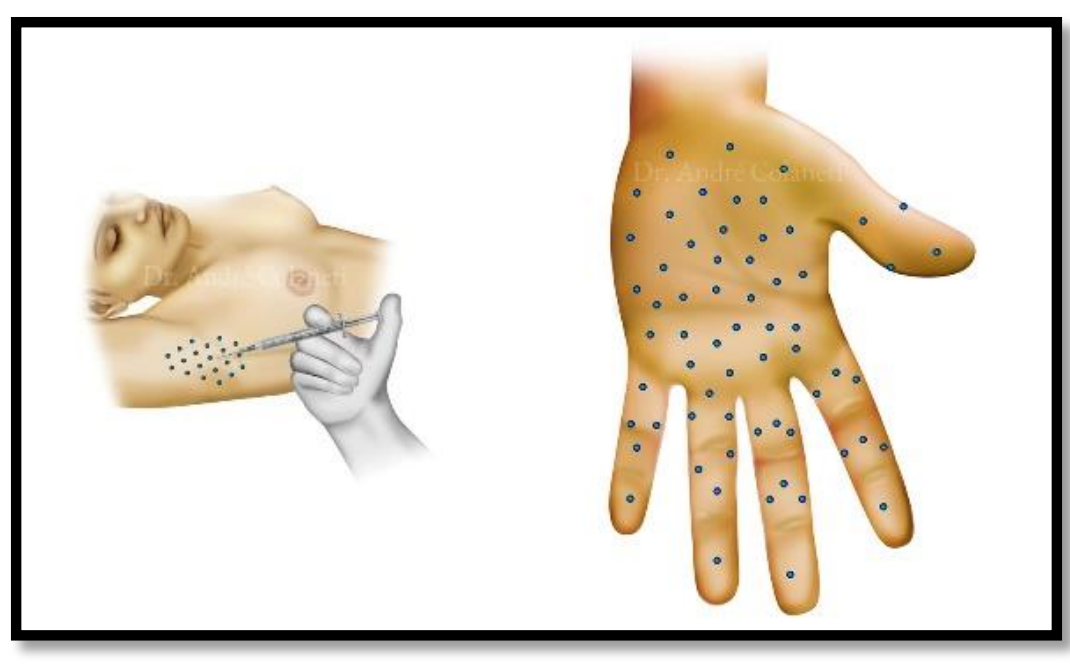

FONTE: https://www.cirurgiaestetica.com.br/botox-para-tratar-hiperidrose/

A injeção de toxina botulínica altamente diluída diretamente na pele das axilas ou na pele das palmas das mãos e solas dos pés pode aliviar a transpiração excessiva. As glândulas sudoríparas da pele ficam paralisadas, causando suor excessivo. Um tratamento pode proporcionar meses de alívio, e os especialistas acreditam que a injeção pode ser repetida indefinidamente, uma ou duas vezes por ano.

\section{ATUAÇÃO DO FARMACEUTICO NA ORIENTAÇÃO AO USO DA TOXINA BOTULÍNICA}

Segundo as resoluções $n^{0}$ 573/13 e nº 616/15, foi estabelecida uma nova área de atuação, permitindo ao Farmacêutico esteta recursos utilizados na saúde estética, como: aplicação de toxina botulínica, preenchimento dérmico, carboxiterapia, intradermoterapia/mesoterapia, agulhamento e microagulhamento estético e criolipólise (ALVES, et al, 2016). 
O Conselho Federal de Farmácia (CFF) esclareceu que, ao contrário do que vem sendo divulgado, os farmacêuticos estão autorizados a atuar na área de estética, inclusive com aplicação de toxina botulínica. A decisão do Tribunal Distrital Federal da Primeira Região que não favorece a atuação do farmacêutico na área da beleza não abrange todos os profissionais.

Esta ação invalidou temporariamente a Resolução 573/13 / CFF porque o CFF já havia apelado da decisão. Outras resoluções CFF 616/15 e 645/17 ainda são válidas, incluindo a aplicação de toxina botulínica, preenchimento cutâneo, carbonoterapia, terapia intradérmica / mesoterapia, agulhas / microagulhas de beleza e criolipólise.

\section{CONCLUSÃO}

Conclui se que realmente a TBA é uma droga com bons recursos terapêuticos e é considerada segura clinicamente. A TBA traz melhora a qualidade de vida, saúde estética e emocional dos pacientes que aderem ao seu uso.

O farmacêutico esteta está autorizado a atuar na área de estética, inclusive na aplicação da toxina botulínica.

\section{REFERÊNCIAS}

RIBEIRO, ISAR. SANTOS, ANA. GONÇALVES, VIRGINIA. CRUZ, EDGAR. O USO DA TOXINA BOTULÍNICA TIPO “A” NAS RUGAS DINÂMICAS DO TERÇO SUPERIOR DA FACE. 2014. 7f. Revista da Universidade Ibirapuera Universidade Ibirapuera São Paulo, v. 7, p. 31-37. Disponível em: http://www.revistaunib.com.br/vol7/o3.pdf Acessado em I6/03/202I.

ALVES, HÉRICK. BATISTA, EMANUEL. RODRIGUES, SUIANI. SILVA, LUANA. FRAGA, EDMIR. ATUAÇÃO DO FARMACÊUTICO NA SAÚdE ESTÉTICA. 2016. 6f. Disponível em: file:///C:/Users/isabe/Downloads/1220-3243-I-PB.pdf Acessado em: I6/03/202I.

SPOSITO, MARIA. Toxina botulínica tipo A - propriedades farmacológicas e uso clínico. 2004. 38f. Disponível em: file:///C:/Users/isabe/Downloads/ro2495Texto\%20do\%2oArtigo-I78995-I-I0-201508I8.pdf Acessado em:I6/o3/2021. 
UEBEL, MARJORIE. USO DA TOXINA BOTULÍNICA NA PREVENÇÃO DE RUGAS DINÂMICAS - UMA REVISÃO DA LITERATURA. 2019. IIf. Artigo apresentado na disciplina de Trabalho de Conclusão de Curso, do Curso de Pós Graduação em Farmácia Estética da Universidade do Vale do Taquari. Disponível em: https://www.univates.br/bdu/bitstream/10737/2631/I/2019MarjorieRoeslerUebel\%20\%28I \%29.pdf Acessado em:16/03/2021.

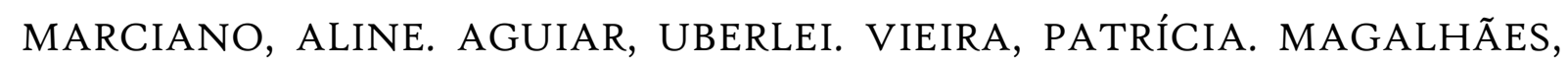
SERGIO.

TOXINA BOTULÍNICA E SUA APLICAÇÃO NA ODONTOLOGIA. IIf. Revista de Iniciação Científica da Universidade Vale do Rio Verde, Três Corações, v. 4, n. I. 2014. Disponível em:file://C:/Users/Isabella/Downloads/I554-4216-I-PB.pdf

https://prodoctor.net/blog/farmaceuticos-estao-autorizados-aplicar-toxina-botulinica/ Acessado em: 20/09/2021.

https://www.cirurgiaestetica.com.br/botox-para-tratar-hiperidrose/ Acessado em: 20/09/2021.

https://www.saude.se.gov.br/farmaceuticos-tem-papel-essencial-para-o-bom-

funcionamento-do-sus/ Acessado em: 20/09/2021.

https://www.sbd.org.br/dermatologia/pele/procedimentos/toxina-botulinica-tipo-a/r3/ Acessado em: 20/o9/2021. 